Document downloaded from:

http://hdl.handle.net/10251/99659

This paper must be cited as:

Benaatou, W.; Latif, A.; Pla, V. (2017). Vertical Handover Decision Algorithm in Heterogeneous Wireless Networks. International Journal of Internet Protocol Technology (Online). 10(4):197-213. doi:10.1007/s11235-017-0331-2

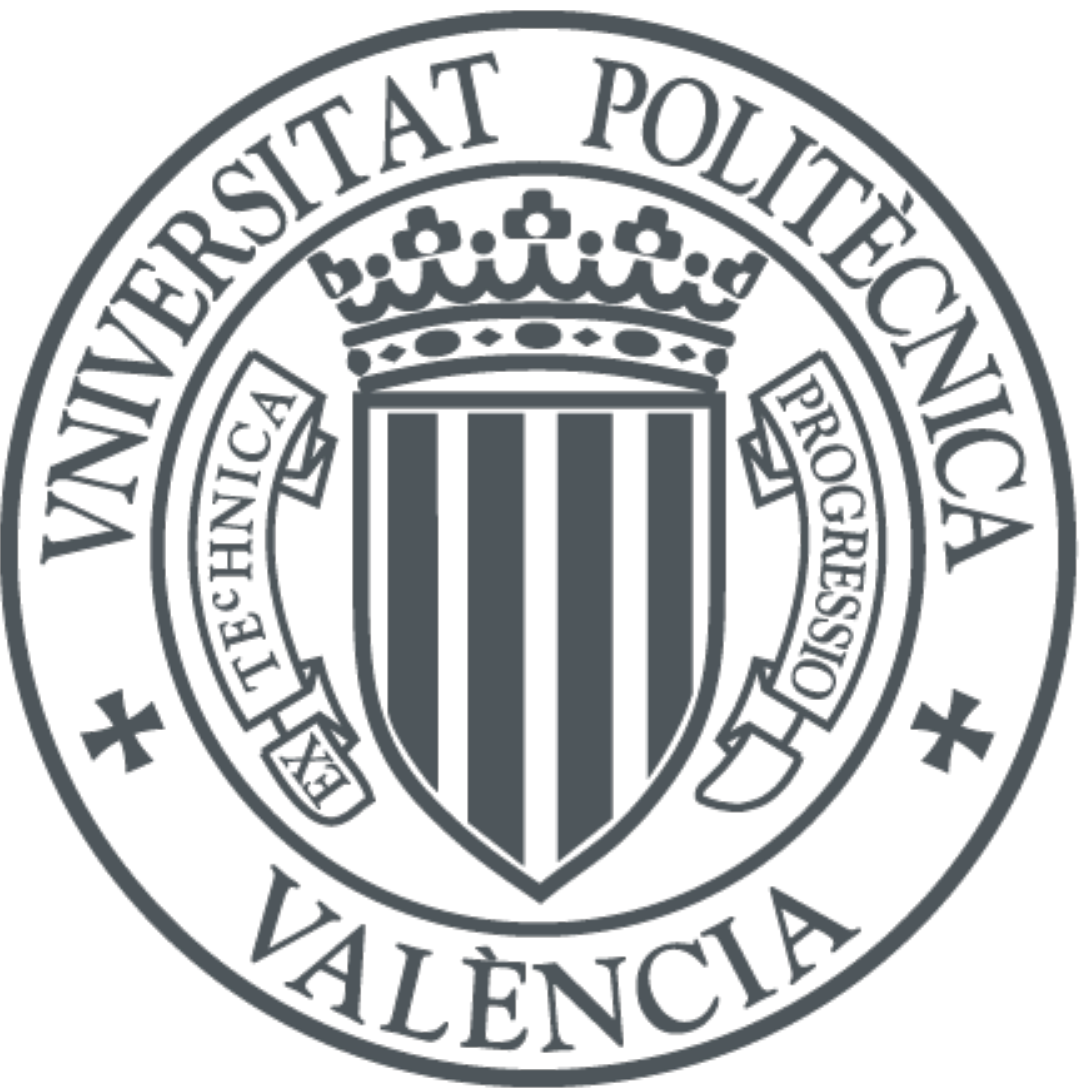

The final publication is available at

https://doi.org/10.1007/s11235-017-0331-2

Copyright Inderscience Publishers

Additional Information 


\title{
Vertical handover decision algorithm in heterogeneous wireless networks
}

\author{
Wafa Benaatou* and Adnane Latif \\ TIM, \\ National School of Applied Sciences, \\ Cadi Ayyad University, \\ Marrakech, Morocco \\ Email:wafaa.benaatou@gmail.com \\ Email: a.latif@uca.ac.ma \\ *Corresponding author

\section{Vicent Pla} \\ ITACA, \\ Universitat Politècnica de València, \\ Camino de Vera s/n, 46022, València, Spain \\ Email:vpla@upv.es
}

\begin{abstract}
With the recent progress in the area of cellular communication the issue of inter cells handover without dropping an on-going connection with the base station has arisen. In this paper, the focus is on the performance of vertical handover. Various proposed interconnection architectures for vertical handover in heterogeneous networks were studied. Two different algorithms to make the decision on when and to which network perform a handover were considered. In the first of them the decision is based on the received signal strength (RSS). In the second one a fuzzy logic system that uses RSS, bandwidth, battery power and packet loss as the input parameters is proposed. The simulation results show that the algorithm based on fuzzy logic leads to a reduction of the number of handovers and a minimization of the power consumption as compared to the first algorithm used here and the existing algorithms.
\end{abstract}

Keywords: vertical handover; wireless local area network; WLAN; WIMAX; UMTS; LTE; LTE-A; received signal strength; RSS; heterogeneous networks; fuzzy logic; simulation.

Reference to this paper should be made as follows: Benaatou, W., Latif, A. and Pla, V. (xxxx) 'Vertical handover decision algorithm in heterogeneous wireless networks', Int. J. Internet Protocol Technology, Vol. X, No. Y, pp.xxx-xxx.

Biographical notes: Wafa Benaatou is a $\mathrm{PhD}$ in Telecommunications at the National School of Applied Sciences, University Cadi Ayyad, Marrakech, Morocco. She received her Master's degree in Software Quality from the Faculty of Sciences-Fes Morocco. During her study period and previous professional training experiences, she was given an effective contact to all the considered fields: computer sciences, decision making, leadership skills, communication and teamwork capabilities, development and programmation. Her current research interests include wireless communications, heterogeneous networks, fuzzy logic, modelling and cellular networks.

Adnane Latif is a PhD in Telecommunications and Networks and Professor at the Cadi Ayyad University, National School of Applied Sciences, Marrakesh, Morocco. His researches in the area are antennas, microwaves and wireless communications. He is an author of a fifties publications in international conferences and in international journals. He is a Founder and Coordinator of the Spring School in 2010 "Wireless and Emerging Technologies". He is a Founder and President of the Moroccan Association of Telecommunications and Electronics Technologies. He is a member organiser of several international conferences and Spring Schools. He is a member of international program committee of several international conferences. He is also a reviewer and board member of several international journals and author of two books.

Vicent Pla received his ME and PhD in Telecommunications from the Universitat Politècnica de València (UPV), Spain, and the BSc in Mathematics from the Universidad Nacional de Educación a Distancia, Spain. In 1999, he joined the Department of Communications, UPV, where he is currently an Associate Professor. His research interests lie primarily in the area of modelling and performance analysis of communication networks. During the past few years, most of his research activity has focused on resource management in wireless networks. In these areas he has authored numerous papers in refereed journals and conference proceedings, and has been an active participant in several national and European research projects. 


\section{Introduction}

Wireless technologies such as WLAN, WiMax, UMTS, LTE and LTE-A were developed with different standards and these technologies offer a variety of services, different data rates and diverse areas of coverage. To provide a better connection between heterogeneous networks it is necessary to perform a handover (HO) either vertical or horizontal. A horizontal $\mathrm{HO}$ is defined as $\mathrm{HO}$ between different base stations (BS) with the same wireless network interface that is started if only the base station is changed, but the technology remains the same (for example, a transfer starting from a cell WLAN1 to a WLAN2). A vertical HO is a $\mathrm{HO}$ between base stations that use different network interfaces (wireless network interface, such as WiMax, WLAN,...) meaning that it is carried out in the case of a change of the network and type of technology (for example, a transfer of a WLAN cell to a UMTS cell). It is this second type of $\mathrm{HO}$ that is the focus of this study. It is for this reason that it will be considered that whenever the term $\mathrm{HO}$ in our paper is used, it will, in fact, refer to the vertical HO.

The HO process is divided into two parts, one is the $\mathrm{HO}$ decision process and the other is the HO execution process. In the $\mathrm{HO}$ decision process both the mobile node and network decides when the $\mathrm{HO}$ process will occur. After the completion of the $\mathrm{HO}$ decision process, the $\mathrm{HO}$ execution process continues. The $\mathrm{HO}$ execution process collects the supplementary network information such as the address detection time in mobile IPv6 and when the HO decision will occur and detect process overlaps. The HO process is required when the following situation occurs: When the motion of the user equipment (UE) is very fast, for example, the movement of the user's equipment from one cell to another during an ongoing session, there will be interference phenomena caused by the user's equipment from the near cell. These are some basic points due to which the network decides that the HO process is required. The main aim of the $\mathrm{HO}$ process is to allow the mobile users to roam freely from one mobile network to another either within the same network or a different one. To achieve the load balancing in the different cell $\mathrm{HO}$ is also required, and it is also necessary to maintain good radio quality of the link between the mobile users and the serving BS and to minimise the interference level.

This paper presents the performance analysis of vertical $\mathrm{HO}$ in heterogeneous networks and focuses mainly on the study of the received signal strength (RSS) in the reach of mobile station using MATLAB. MATLAB was used because it offers a wide range of possibilities for simulation of different wireless networks and the key contribution of this work concerns the implementation of the HO between five types of access networks: (WLAN, UMTS, WiMax, LTE and LTE-A) in order to provide a seamless vertical HO across heterogeneous access networks such as these The RSS in the proposed interconnection architectures was implemented mostly between LTE and LTE-A networks and several parameters (RSS, bandwidth, battery power and packet loss) were considered, using fuzzy logic to make the decision on when and which network to perform a $\mathrm{HO}$ to.
Moreover, the vertical HO decision (VHD) based on RSS only, the VHD based on fuzzy logic and the other VHD algorithms were compared. The rest of the paper is organised as follows. Section 2 describes the vertical HO procedure. Section 3 describes the related work. Section 5 presents the simulation environment with results and discussion. Finally, Section 6 concludes the paper.

\section{Vertical HO procedure}

A vertical $\mathrm{HO}$ can be divided into four steps:

- determination of the surrounding systems

- analysis of the quality of service (QOS) available on the detected systems

- determination and selection of the most appropriate network

- implementation of the vertical HO.

All these steps must proceed so as to ensure a vertical HO without cuts and must be proactive. 'Proactive', expresses the idea that the client must be able to precisely obtain user expectations in terms of QOS and the performance, in order to make the best possible decision.

\subsection{Determination of active systems in the radio environment}

This is the first step of the vertical HO, and is crucial. Thanks to this step, the cognitive terminal can determine the list of candidate networks for the vertical HO. Indeed, once the terminal has successfully detected the presence of a signal, it would know to which system the detected signal belongs. This identification must be done without the terminal attempting successive connections to each of the active systems.

\subsection{Analysis of the quality of discovered systems}

Once these systems have been identified, the terminal undertook a series of measures regarding the link quality that can be attained in each network. The horizontal HO based on the metric of the received signal power generally is triggered. Unfortunately, this metric is not sufficient to trigger a vertical $\mathrm{HO}$.

To find the best system, the $\mathrm{HO}$ procedure requires more information and parameters in order to decide to which system it must switch to. This information and parameters are more commonly known under the name of 'metric of HO'. They are parameters measured by the terminal in order to give an indication on the feasibility of the vertical $\mathrm{HO}$ and to help with the choice of the target system. Besides the signal report with noise, for a vertical HO there are additional parameters to take into account, for example:
Comment [a1]: Author: Please confirm if the amended changes are correct. 
- Static user preferences that refer to a list of services to which the user has subscribed, and a preference list indicating the priority of services in case of scarce resources.

- Dynamic measures that contain a list of supported or unsupported services on the network; a list of active or suspended services; and an indicator on the QOS delivered. These parameters include the monitoring and analysis of networks such as the reception power settings, bit error rate, the block error rate, the effective rate, the battery status, the coverage area, the access cost, security and services available.

\subsection{Decision and selecting the most appropriate network}

Once vertical HO metrics have been identified, they are collected in a measurement report and transmitted to the upper layers, i.e., to the entity in charge of the HO decision, to make a decision. This decision is based on the QoS requirements of the application used by the user. Once the decision is made and the target network is selected, the execution procedure for the vertical $\mathrm{HO}$ is triggered. Note the $\mathrm{HO}$ can be executed by the mobile, which is known as mobile-controlled HO (controlled terminal mode), or may arise from a decision-making entity of the network, in this case known as network-controlled HO (controlled network mode), or even jointly in cooperation with both, then this is the mobile-assisted HO (user controlled network and assisted terminal). Controlled terminal mode is a decentralised decision. While the network mode is controlled, it is mainly used in circuit-switched networks. The advantage of this mode is that the network monitors its load and can avoid saturation of resources that cannot be controlled in terminal mode. Finally, the controlled network mode is used by terminal served by the UMTS network.

\subsection{Implementation of vertical $\mathrm{HO}$}

Once the network targets have been selected, the entity responsible for execution, terminal or network, is informed of the HO to be achieved. Thus, connection needs to be switched from the current network to the selected network in the most available transparent way.

This last stage includes also the stages of authentification, authorisation and transfer of identity of the user towards the new access point (AP). The fact that the terminal must be able to rock from one network to another means that the terminal must be equipped with a flexible radio architecture which enables it to change its connection parameters in order to adapt to the network chosen by the vertical HO process.

The HO mechanisms have a four different phases: HO initiation, system discovery, $\mathrm{HO}$ decision, and $\mathrm{HO}$ execution as illustrated in Figure 1.
Figure 1 HO phases (see online version for colours)

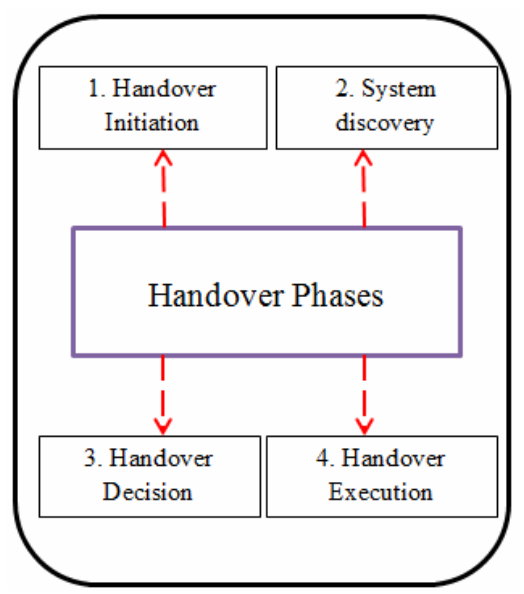

\section{Related work}

Many VHD algorithms have been proposed in the research literature, most of them have designed their VHD algorithms depending on the signal strength received by the mobile terminal, where $\mathrm{HO}$ decisions are made by comparing the received signal strength with the threshold values. These algorithms which use signal strength as their basic HO decision indicator are called RSS algorithms. However, the data rate achieved by a mobile terminal is related to its signal to interference and noise ratio (SINR), which is a function of the interference in the network, as well as the distance between BS and AP to the mobile terminal. RSS based VHD occurs when the mobile terminal receiving power approaches the threshold value regardless of the QoS needed, thus rendering RSS-based VHD not to support user's QoS requirement. On the other hand, SINR based VHD supports multimedia QoS requirement depending on the achievable data rate which leads to seamless vertical HO.

1 "RSS measurement for vertical handoff in heterogeneous network" by Ayyappan and Dananjayan (2008). In this paper, a method is proposed to evaluate the performance of vertical $\mathrm{HO}$ in terms of received signal strength measurement using suitable propagation model for heterogeneous network employing cellular, WLAN and HIPERLAN.

2 "A study of RSS based vertical handover decision algorithms" by Gavali and Patil (2015). This paper represents the RSS based VHD algorithms and a comparison of these VHD algorithms.

3 "A smart triggering scheme to reduce service interruption during heterogeneous handovers" by Huaiyu et al. (2008). In this paper, a smart triggering scheme was proposed, which based on received signal strength indication (RSSI) predication. The RSSI could vary when the $\mathrm{MN}$ moves, due to the effect of
Comment [A2]: Author: Please provide full reference or delete from the text if not required. 
shadowing and fading. An exponential average of smoothing predication method was adopted to predicate the RSSI. The link status would be recorded and compared with the trend of RSSI. When the predicated RSSI is below the predefined link going down (LGD) threshold, and the long-term trend of RSSI is going downward, a pre-trigger event will be generated.

4 "Predictive link trigger mechanism for seamless handovers in heterogeneous wireless networks". Here, another RSSI-based predicative link trigger mechanism was proposed. The HO procedure may fail due to too early or too late to trigger. The required $\mathrm{HO}$ time is estimated at first, and then a predicative link trigger mechanism is executed once the filtered sample power is less than a predefined predication start threshold. The threshold is determined by the required $\mathrm{HO}$ time. If the value is less than the minimum power level, the $\mathrm{HO}$ procedure is initiated.

5 "Algorithmic Vertical Handoff decision and merit network selection across heterogeneous wireless networks” by Kunarak and Suleesathira (2013). In this paper the RSS is predicted by back propagation neural network which is beneficial as it performs the handoff early. Dwell time value depends on the user speed and moving pattern. The policy for triggering a handoff is that the RSS conditions are consistently true during dwell time, so that unnecessary handoffs are avoidable.

6 "An adaptive vertical handoff algorithm based on UMTS and WLAN" by Ling et al. (2014). In this paper a vertical handoff (VHO) algorithm based on UMTS and WLAN integration framework is proposed in order to make it more effective, reduce the ping-pong effect and interruption probability.

7 "IEEE 802.21 based vertical handover in WiFi and WiMax networks” by Bathich et al. (2012). This paper evaluated the SINR based vertical $\mathrm{HO}$ algorithm and RSS based vertical HO algorithm in terms of the maximum downlink throughputs. The Shannon capacity determines the maximum achievable data rate for a given SINR and carrier bandwidth.

8 "Combined SINR based vertical handoff algorithm for next generation heterogeneous wireless networks" by Yang et al. (2007). This paper proposes a novel VHO algorithm which uses received SINR from various access networks as the handoff criteria. This algorithm considers the combined effects of SINR from different access networks with SINR value from one network being converted to equivalent SINR value to the target network, so the handoff algorithm can have the knowledge of achievable bandwidths from both access networks to make handoff decisions with QoS consideration.

9 "A combined vertical handover decision metric for QoS enhancement in next generation networks" by Vegni et al. (2009). This proposed combined VHO approach contains both RSS and SINR parameters are in terms of both end user efficiency, i.e., cumulative received bits, and network performances, i.e., VHO improves QOS for mobile users, a data rate gain parameter.

10 "Distance-based scheme for vertical handoff in heterogeneous wireless networks” by Mardini and Al-Ghadi (2012). This paper proposes a distance-based scheme for vertical handoff (DSVH) for heterogeneous wireless networks. The main goal of this scheme has been achieved. It is to enhance and provide higher overall system performance in terms of minimising service disconnection probability during $\mathrm{VHO}$ as compared to the SINR based VHO scheme.

11 "Signal strength ratio based vertical handoff decision algorithms in integrated heterogeneous networks" by Sanjay and Reddy (2014). This paper proposed the signal strength ratio (SSR) based VHO algorithms and the average received signal strength (ARSS) based VHO algorithms for integrated networks of wireless local area network (WLAN) and 3G networks and evaluated the performance of the proposed VHO algorithms in terms of the number of handoffs and decision delay.

12 "Predictive RSS with fuzzy logic based vertical handoff algorithm in heterogeneous wireless networks" by Kunarak and Suleesathira (2010). This paper proposes a predictive RSS and fuzzy logic based network selection for VHO in heterogeneous wireless networks. The RSS predicted by back propagation neural network is beneficial as it avoids dropping calls if it predicts a mobile is moving away from the monitored wireless network. In addition to the RSS metric, the residence time in the target network is predicted which is taken into account for the handoff trigger.

13 "Fuzzy multi-parameter based vertical handoff decision algorithm”. In this paper Bin et al. (2013) proposed a fuzzy multi-parameter based VHO decision algorithm this study considers five parameters of network and user, and applies a multi-mode smart terminal based speed adaptive VHO policy to improve the network update speed and lower network blocking probability.

14 "A fuzzy logic approach for quality of service quantification in wireless and mobile networks". In this paper, Farnaz et al. (2014) evaluated a method using fuzzy logic to improve the quantification of QoS level for both homogeneous and heterogeneous networks.

15 "Fuzzy logic based layers 2 and 3 handovers in IEEE 802.16e network”. In this paper, Bchini et al. (2010) studied a fuzzy logic based scheme for fast selection of best $\mathrm{BS}$ and of $\mathrm{HO}$ technique at the $\mathrm{HO}$ time in order to minimise the delay during $\mathrm{HO}$ for sensitive multimedia traffic.

16 "Complexity-consistency trade-off in multi-attribute decision making for vertical handover in heterogeneous wireless networks". In this paper, Chinnappan and
Comment [A3]: Author: Please provide full reference or delete from the text if not required. 
Balasubramanian (2016) used the fuzzy logic to estimate the necessity of $\mathrm{HO}$ and to determine the user satisfaction degree based on critical parameters, such as mobile terminal speed, network load and service cost.

17 "A fuzzy logic-based communication medium selection for QoS preservation in vehicular networks". In this work Bouali and Senouci (2016) developed a fuzzy based framework to select the best communication medium in a heterogeneous vehicular network and considers several features that affect the decision process, which are available from the network such as received signal strength (RSSI), network density, vehicle speed and service cost.

\section{Results and discussion}

\subsection{HO performance in heterogeneous networks}

Mechanism of HO has four different phases: initiation of HO; HO decision making; selection of network; and execution of HO process. HO decided by QOS parameter, such as strength of the signal and quality of the network link, etc., is known as initiation of HO. In HO decision making, the signal strength and the QOS parameters of the neighbouring networks are measured and a decision is taken to select the best network suitable to perform the HO. Network selection phase identifies the best suitable network among all the available networks chosen to perform the HO. Execution of $\mathrm{HO}$ deals with establishment of connection, release of connection, and network security aspects as show in Figure 2.

Figure 2 Mechanism of HO vertical in wireless networks (see online version for colours)

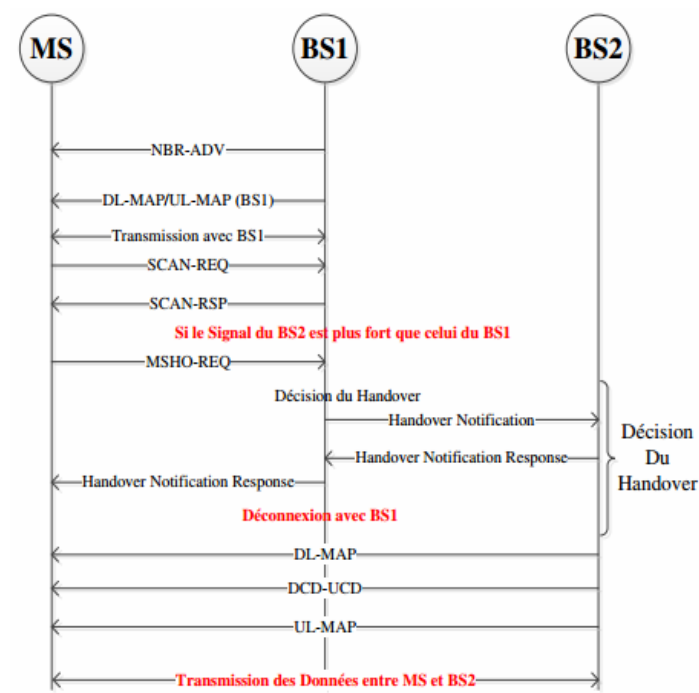

In WLAN there are two reasons to run a HO: the signal level and the traffic load. Signal level: if the signal level provided by a base station is no longer satisfactory, a HO onto another base station is initiated if it provides a higher signal level. Traffic load: due to the traffic load, a BS cannot provide bandwidth and optimal service quality.

Figure 3 Proposed interconnection architecture of WLAN and UMTS (see online version for colours)

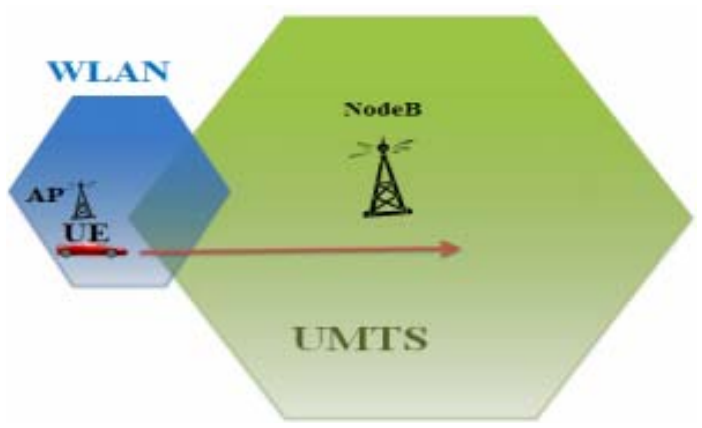

In the UMTS, HO is performed if the signal level reaches a certain threshold value specified by the radio network controller (RNC). It can be applied for the uplink and downlink it can also be implemented if the cell traffic becomes too heavy. HO in UMTS still depends on the user's mobility, traffic distribution, bandwidth and change of service.

Figure 4 Proposed interconnection architecture of WLAN and WiMax (see online version for colours)

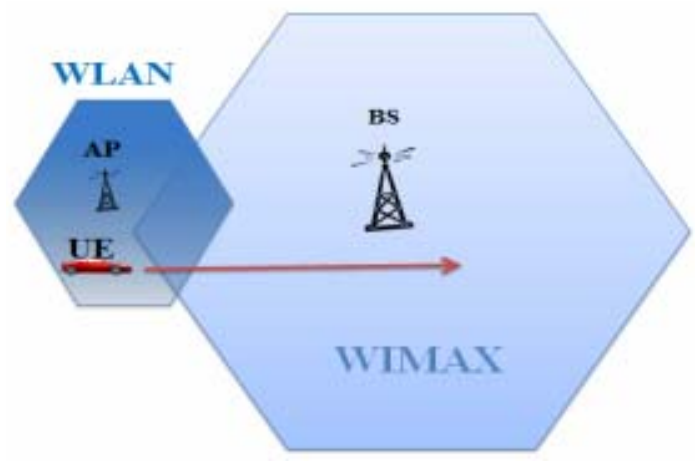

Figure 5 Proposed interconnection architecture of WiMax and UMTS (see online version for colours)

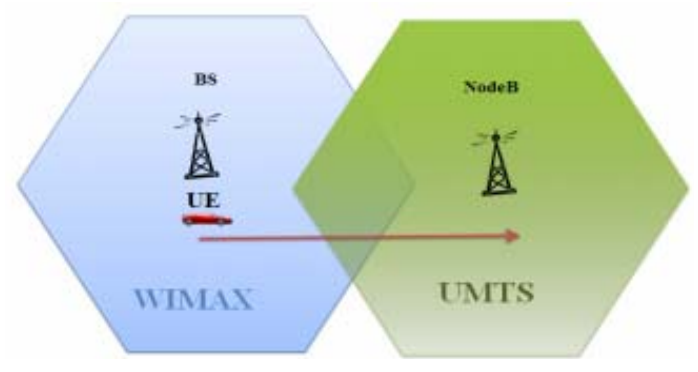

In WiMax, the decision for the HO can be started by the MS or by BS. During the process of $\mathrm{HO}$, the MS has the right to 
cancel the procedure and to recommunicate again with the previous ones.

In the LTE, the HO is started by the UE, who sends a measurement report to the e-NodeB source, this last will decide to carry out the $\mathrm{HO}$ or not, the e-NodeB source makes its decision based on the report of level of power received.

Figure 6 Proposed interconnection architecture of multi WLAN and UMTS (see online version for colours)

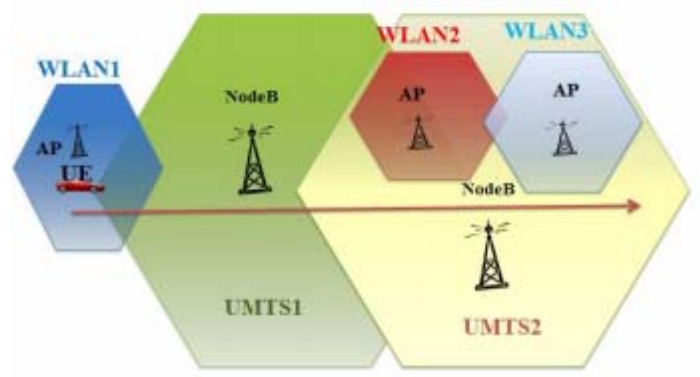

To illustrate our simulation, interconnection architecture between different networks has been designed:

- Figure 3. Initially a situation according to which a mobile station would move at constant speed since a WLAN network towards nearby networks UMTS was imagined.

- $\quad$ Figure 4. Secondly, a situation according to which a mobile station would move at constant speed since a WLAN network towards nearby networks WiMax was imagined.

- $\quad$ Figure 5. As can be seen, in this proposed interconnection architecture, the trajectory of station mobile is from WiMax to UMTS.

- Figure 6. This interconnection architecture shows the HO between multi-WLAN and UMTS and the MS moves from WlAN1 to UMTS2.

- $\quad$ Figure 7. In this interconnection a situation according to which a mobile station would move at constant speed since a WLAN network towards networks nearby WiMax and UMTS was imagined.

- $\quad$ Figure 8. In this proposed interconnection a situation in which a mobile station would move at a constant speed from a WiMax to a neighbouring LTE network was imagined.

- $\quad$ Figure 9. This simulation represents the combination of three technologies WLAN, WiMax and LTE. The movement of the mobile station from a WLAN network to a neighbour network WIMAX and LTE.

- Figure 10. The last simulation represents the combination of LTE and LTE-A. The movement of the mobile station from a LTE network to a neighbour network LTE-A.

\subsection{HO decision based on RSS}

There follows a simulation of the $\mathrm{HO}$ situations, in particular HO based on the strength of the signal received (RSS). The proposed algorithm is aimed at vertical HOs in heterogeneous networks. It makes the VHD from the first network to second network taking into account the RSS of the mobile station during its movement.

Figure 7 Proposed interconnection architecture of WLAN, WiMax and UMTS (see online version for colours)

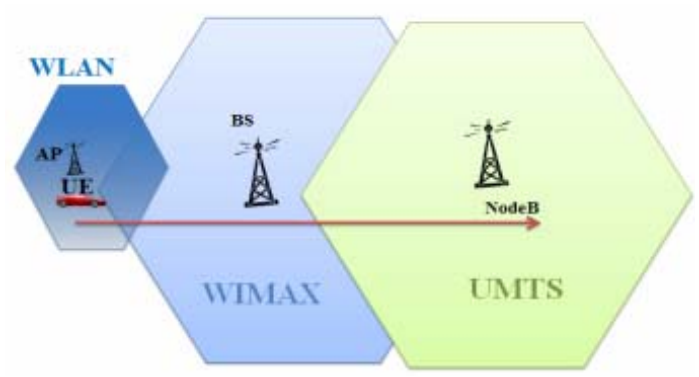

Figure 8 Proposed interconnection architecture of LTE and WiMax (see online version for colours)

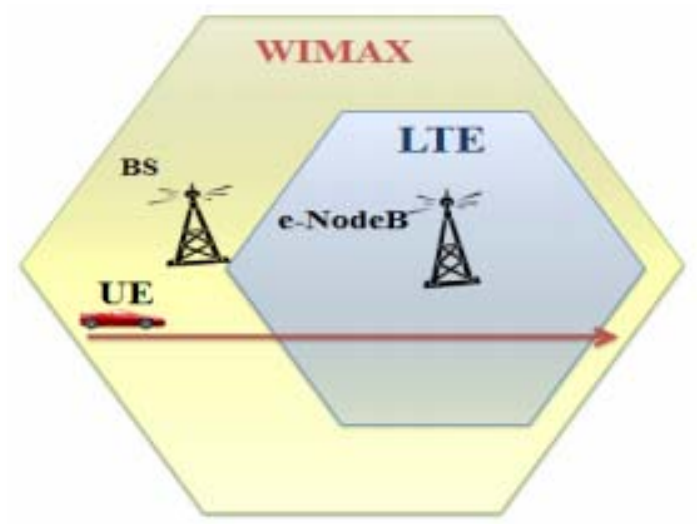

Figure 9 Proposed interconnection architecture of LTE and WiMax (see online version for colours)

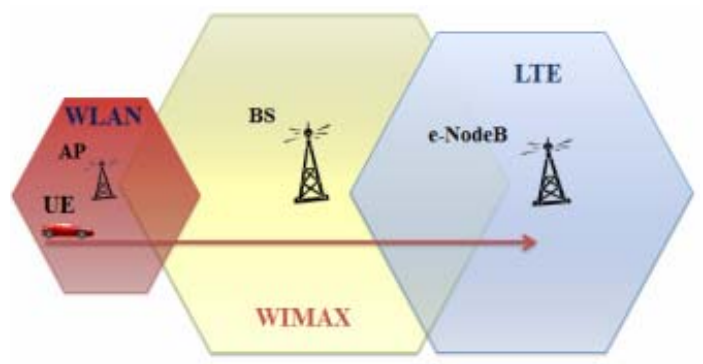

The whole process of decision the $\mathrm{HO}$ is illustrated in Figure 11. This algorithm is in charge of making a decision about when and where to trigger the HO. This decision 
should consider several parameters in order to choose the best candidate network.

This implementation consists of a main program 'main.m' which will call upon 11 other functions that are defined to carry out various tasks: calculation of the slow/fast fading, calculation of the shadowing, and the functions checking if one is in the coverage area of network WLAN, WiMax, UMTS, LTE or LTE-A, these expressions are defined in (1), (2), (3) and (4). The file 'main.m' includes the most important parts of the implementation process, the initialisation of the values of frequencies, the required conditions for the implementation of $\mathrm{HO}$, the speed of the mobile station, etc.

Figure 10 Proposed interconnection architecture of LTE and LTE-A (see online version for colours)

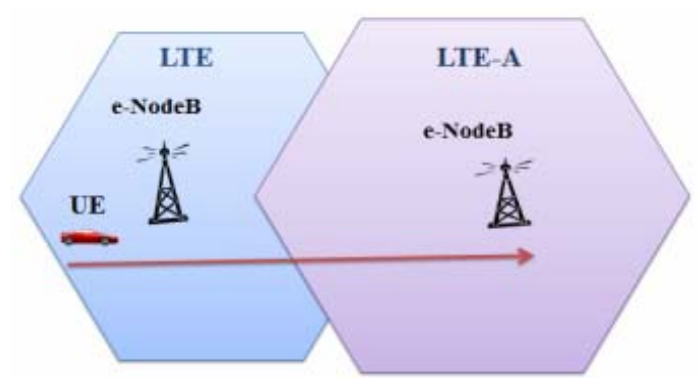

The parameters of simulation of the HO in five networks are illustrated in Table 1.

The general formula of computation applied in the programs is:

$$
R S S=P_{t}-\left(F_{\text {slow }}+F_{\text {fast }}\right)
$$

where $P_{t}$ is the transmitted power and the slow and fast fading are defined by:

$$
F_{\text {slow }}=P L+F_{\text {shadow }}
$$

where $F_{\text {shadow }}$ denotes shadow fading.

$$
P L=P L\left(d_{0}\right)+10 \log \left(d / d_{0}\right)+X_{\sigma}
$$

where $P L\left(d_{0}\right)$ the path loss propagation model, $n$ is the path loss exponent, $d$ is the separation between transceiver and MS in meter. $X_{\sigma}$ is a zero means Gaussian distributed random variable with standard deviation $\sigma$. The reference distance $d_{0}$, the path loss exponent $\mathrm{n}$ and the standard deviation $\sigma$, statistically describes the path loss for an arbitrary distance between transceiver and MS.

$$
F_{\text {fast }}=10 \log \left(E\left(|R(t, k)|^{2}\right)\right)
$$

where $E\left(|R(t, k)|^{2}\right)$ is the mean value of $R(t, k)$. All this gave rise to the results below.

Figure 11 Proposed VHD algorithm (see online version for colours)

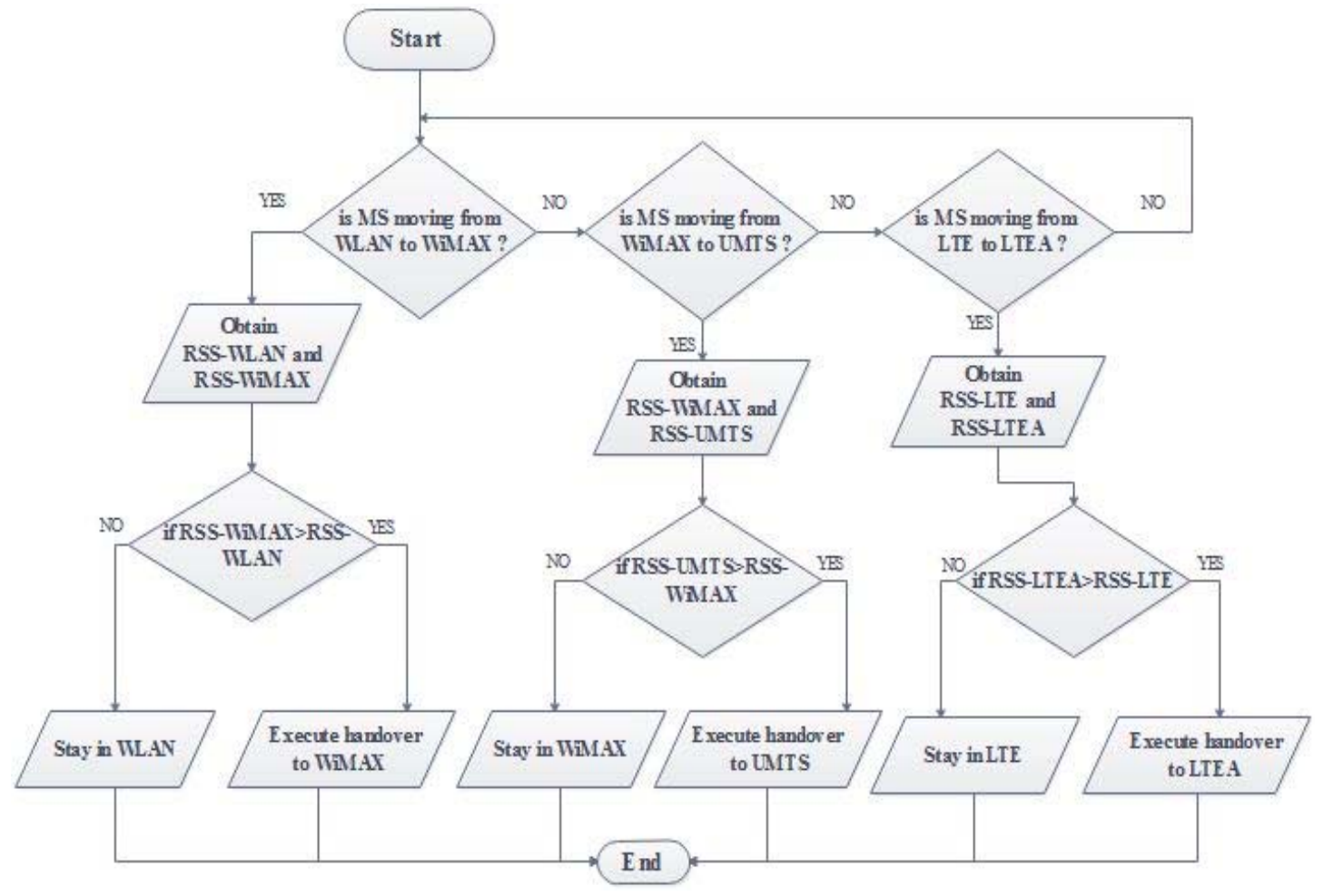


Figure 12 RSS between UMTS and WLAN (see online version for colours)

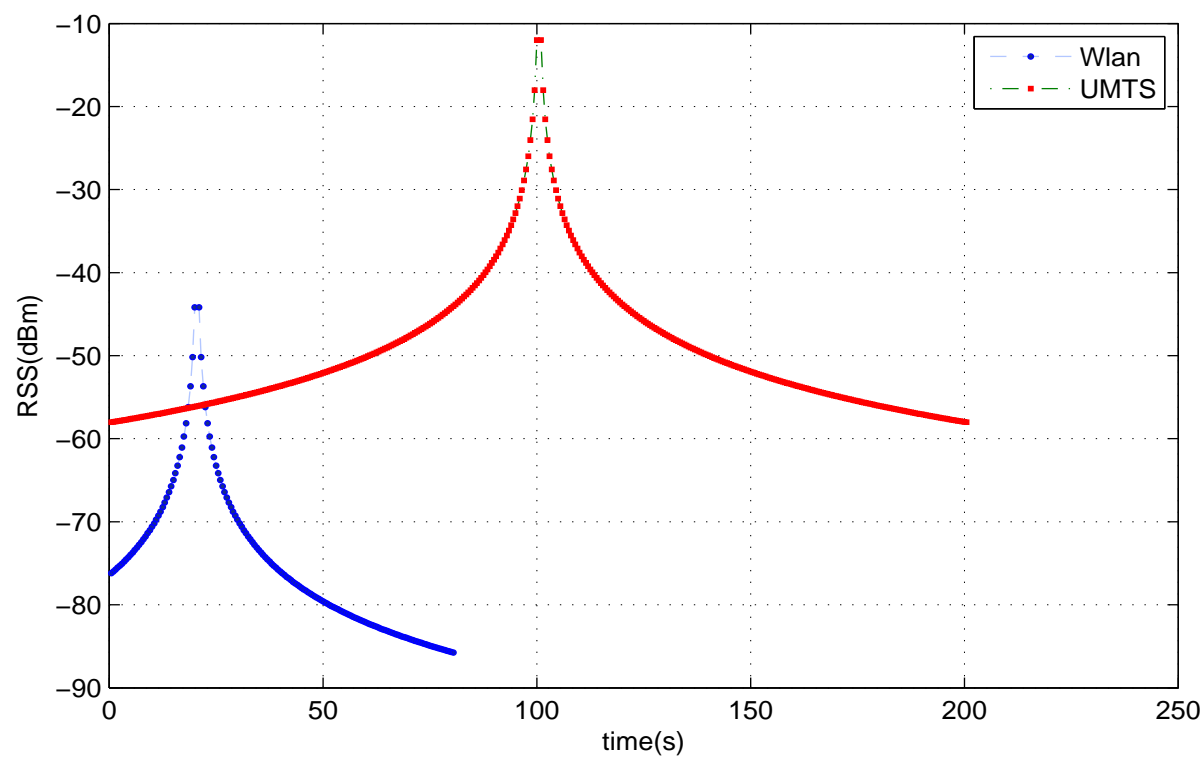

Figure 13 RSS between WLAN and WiMax (see online version for colours)

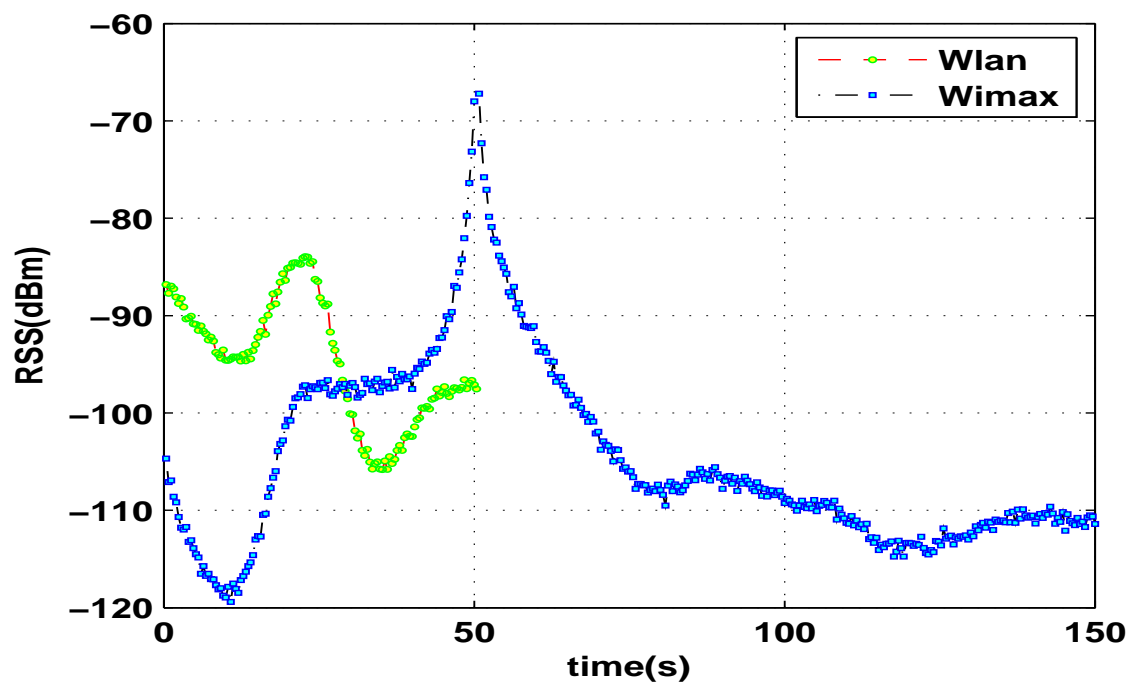

Table 1 Parameters of simulation

\begin{tabular}{lccccc}
\hline \multirow{2}{*}{ Parameter } & \multicolumn{3}{c}{ Value } \\
\cline { 2 - 5 } & LTE $(3 G P P)$ & LTE-A (IMT) & WiMax IEEE 802.16e & WLAN IEEE 802.1 lg & UMTS WCDMA \\
\hline Bandwidth & $10 \mathrm{MHz}$ & $100 \mathrm{MHz}$ & $5-6 \mathrm{GHz}$ & $20 \mathrm{MHz}$ \\
Interval & 0.4 second & 0.4 second & 0.4 second & 0.4 second & $0.4 \mathrm{~second}$ \\
Frequency & $2.6 \mathrm{GHz}$ & $3.7 \mathrm{GHz}$ & $3.5 \mathrm{GHz}$ & $2.4 \mathrm{GHz}$ & $2.1 \mathrm{GHz}$ \\
Speed of mobile station & $10 \mathrm{Km} / \mathrm{h}$ & $10 \mathrm{Km} / \mathrm{h}$ & $10 \mathrm{Km} / \mathrm{h}$ & $10 \mathrm{Km} / \mathrm{h}$ & $10 \mathrm{Km} / \mathrm{h}$ \\
\hline
\end{tabular}


Figure 14 RSS between WiMax and UMTS (see online version for colours)

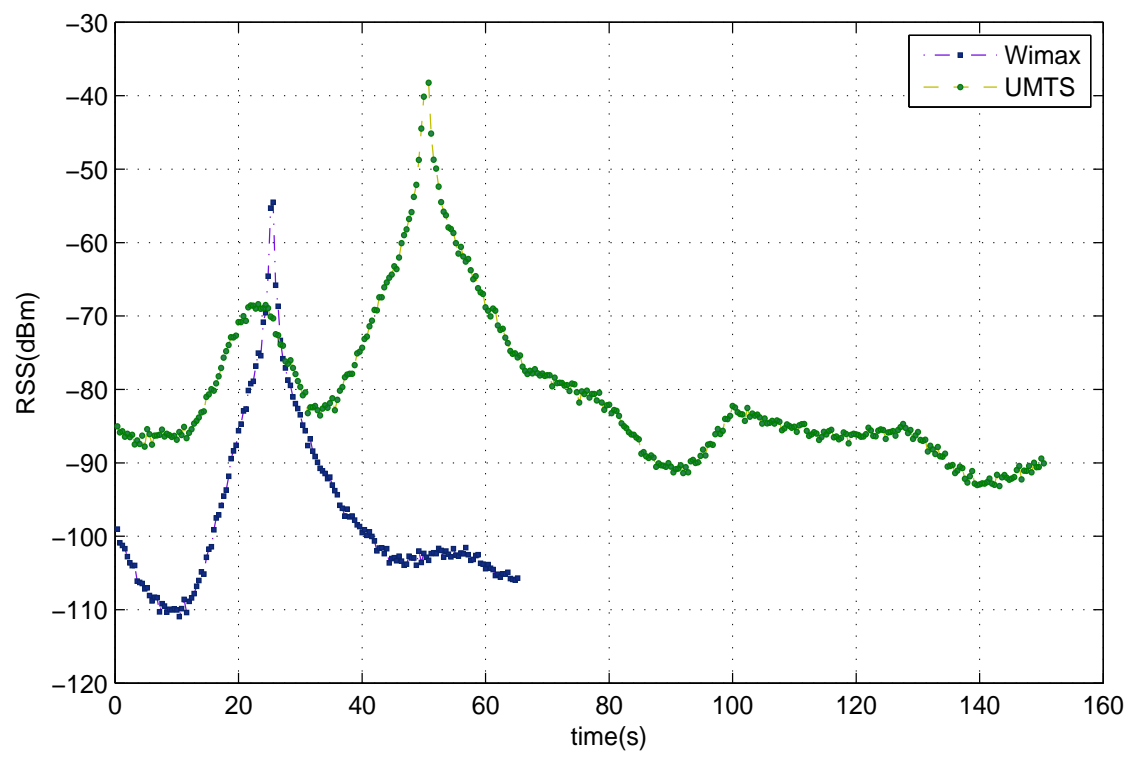

From Figure 11, it is noted that before the significant time $\mathrm{t}=20 \mathrm{~s}$ the station is still connected to NodeB of UMTS because its received power is still greater than the power received at the AP of WLAN. After time $t=20$ s the power received by the AP becomes greater than the power received by NodeB. Therefore, the first $\mathrm{HO}$ is carried out immediately after $t=20$ s, the MS continues to connect to the AP of WLAN to the second significant time $\mathrm{t}=40 \mathrm{~s}$, after which time the power received by NodeB becomes greater than power received by the AP, therefore, the second HO takes place and the mobile station is connected to NodeB of UMTS.

The following simulation of Figure 12 contains two networks WLAN and WiMax. The WiMax station was located much closer to that of WLAN, so a one HO is made of WLAN to WiMax at $t=40$ s when the MS disconnects from the AP of WLAN and connects to BS of WiMax.

In Figure 13, the networks WiMax and UMTS complete the simulation, but by taking into account, at this stage, the effects of the fast fading, as well as shadowing.

These results are slightly different from the first implementation. Indeed this time, the WiMax station was placed much closer to that of UMTS. Thus, a 1st HO is made from WiMax to UMTS, and then at some point, the mobile is replaced in its original configuration where an $\mathrm{HO}$ WiMax to UMTS, always through power measurements, made at intervals.

Figure 14 shows the simulation of two UMTS networks placed several hundred meters away from each other, and functioning on different frequencies. Three WLAN networks were placed a short distance away in such a way as to allow a certain coverage zone of the WLAN and UMTS networks higher definition.
Furthermore, it was decided this time to make a movement of the mobile station which is quasi-random but always towards an adjacent network.

Figure 15 RSS about heterogeneous networks (see online version for colours)

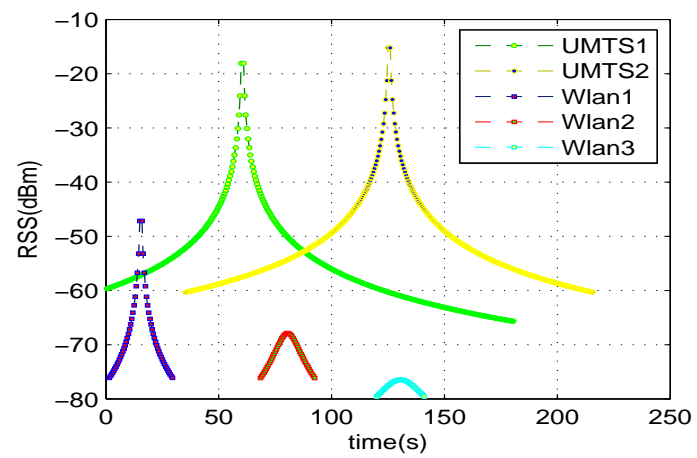

Figure 15 shows the model illustrated in Figure 6, the trajectory of the mobile station is from WLAN1 to UMTS2 and the mobile station uses WLAN1, UMTS1 and UMTS2 which depends on the signal strength. It is clear from the results that the first $\mathrm{HO}$ took place at $\mathrm{t}=20 \mathrm{~s}$ when the signal strength of WLAN1 network is lower than the UMTS1 network. As the MS moves away, the signal strength goes on decreasing from higher value of $-20 \mathrm{dbm}$, therefore the second $\mathrm{HO}$ is executed at $\mathrm{t}=80 \mathrm{~s}$, and the mobile station is still connected to the Node B of UMTS2. 
For the rest, the same calculating made functions are applied, each time with particular regard to slow and fast fading, shadowing, calculation of cover, etc.

In addition, there are times chosen for a displacement of the mobile station which would be quasi-random, but always in direction of an adjacent network.

Figure 16 RSS between WLAN, WiMax and UMTS (see online version for colours)

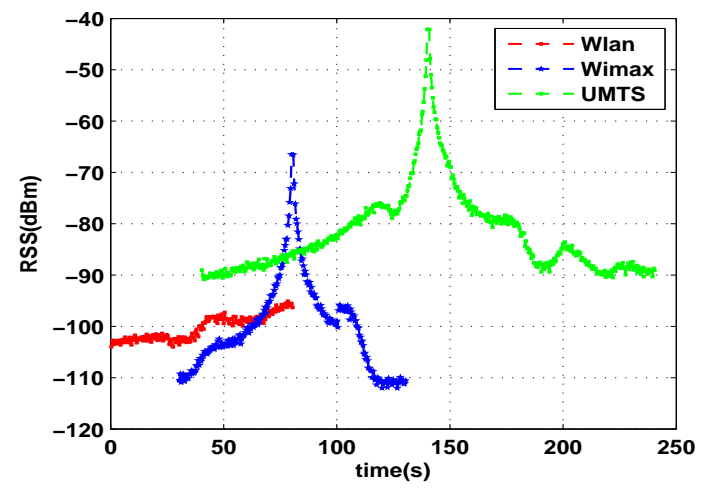

Figure 16 shows the simulation of three networks WLAN, WiMax and UMTS. The 1st HO is made from Wlan to WiMax at $t=60$ s when the MS disconnects from the AP of WLAN and connects to BS of WiMax as the MS moves to UMTS the second $\mathrm{HO}$ is carried out at $t=70 \mathrm{~s}$ when the MS connects to Node B of UMTS and the received signal of UMTS becomes greater than WiMax.

Once the network receives a powerful signal, the mobile station carries out a $\mathrm{HO}$ in order to keep a maximum reception power regardless of its different positions. Whatever the case, it is clear that WLAN does not really constitute a mobile network when compared to WiMax and UMTS. The WLAN covering power remains lower.

Figure 17 RSS between LTE and WiMax (see online version for colours)

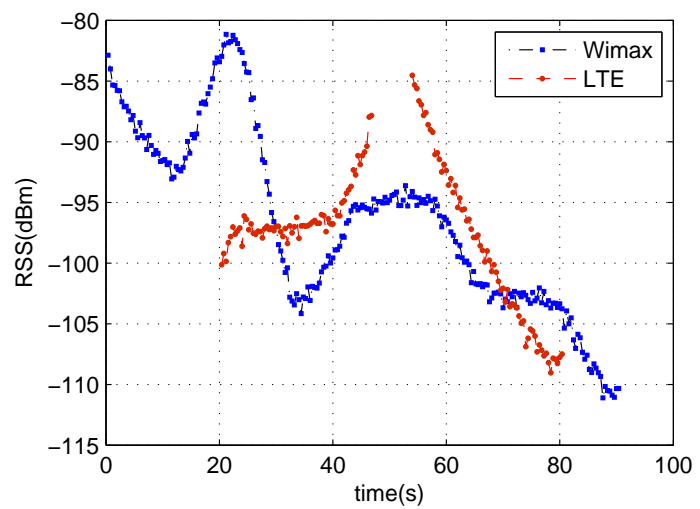

In Figure 17, the RSS between LTE and WiMax according to time is shown. The blue curve represents the power of the WiMax radio link and the red curve shows the power of the
LTE radio link. From the figure, it can be seen that the power of the WiMax radio link decreases gradually as MS moves and enters the overlap area. Then, a new link is detected and the MS starts the first vertical HO process at $\mathrm{t}=30 \mathrm{~s}$, while the second $\mathrm{HO}$ starts at $\mathrm{t}=70 \mathrm{~s}$, especially in the midpoint of the overlapping area, where the RSS from BS is pretty low.

Figure 18 RSS between WLAN, WiMax and LTE (see online version for colours)

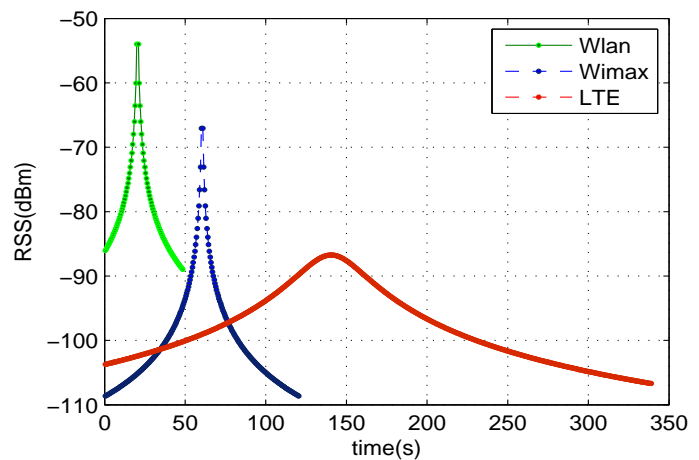

In Figure 18, RSS between WLAN, WiMax and LTE according to time. Each colour refers to one of the networks mentioned above, and here are the representations of the different measurements made by the mobile station as it moves along its path. Each of the above networks has been at one time or another dominant network diagram. Of course, every time a network has benefited from the strength of the stronger signal, the mobile station has performed a HO to keep maximum signal strength in different positions.

Figure 19 RSS between LTE and LTE-A (see online version for colours)

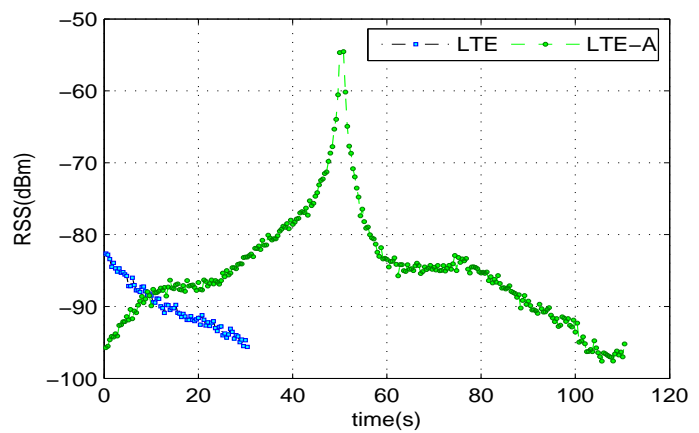

In Figure 19, the RSS between LTE and LTE-A according to time is shown. The blue curve represents the power of the LTE radio link and the green curve shows the power of the LTE-A radio link. From the figure, it can be clearly seen that at a time $t=10$ s the RSS from LTE-A exceeds that from LTE, thus at this moment a HO from LTE to LTE-A will be inititiated. 


\subsection{HO decision based on multi criteria-approach using fuzzy logic}

In this approach four values are considered to make the HO decision; the first value is RSS, the second value is bandwidth, the third value is battery power and fourth value is packet loss. Consequently, the fuzzy system will require these four values as inputs, to obtain one output value (weight value). The Weight value obtained from the fuzzy system is used to enable the system to make a decision to perform the HO between different networks based on the input values.

\subsubsection{HO decision controller}

In this part of the paper fuzzy tool box and Simulink have been used to perform the simulation. The whole process can be explained briefly as:

Fuzzy logic quantative decision algorithm (FQDA), which is an algorithm used widely in VHDs because of its simplicity. Fuzzy logic interference system (FIS) is a part of FQDA. The FIS contains number of functional blocks that are explained as:

- Fuzzifier: input values are converting in the form of membership functions with the help of fuzzifier. The range for these membership functions are also specific to fuzzifier.

- Fuzzy rule database: this defines the set of if-then rules that were useful one in decision making criteria for the HO. The number of rules depends upon the number of parameters used and the set of membership functions.

Rules can be calculated with the following expression or formula:

$[x]^{m}$

where $x=$ number of sets and $m=$ number of parameters.

- Defuzzifier: this block converts the membership value into the single output value. There are two types of FIS: Mamdani FIS and Sugeno FIS.

In this paper Mamdani type FIS is used and the block diagram of basic FQDA is shown in Figure 20.

Figure 20 Block diagram of the fuzzy logic system (see online version for colours)

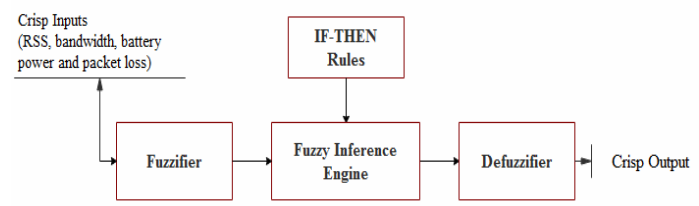

Four parameters have been proposed. Some of parameters depend upon MT and some depends upon the network conditions. The threshold values for each parameter are explained in Table 2.
Table 2 Parameters of simulation

\begin{tabular}{lccc}
\hline Metrics & Low & High & Unit \\
\hline RSS & -100 & -70 & $\mathrm{dBm}$ \\
Bandwidth & 0 & 60 & Bits/s \\
Battery power & 0 & 5 & Watts \\
Packet loss & 0 & 54 & Packets/s \\
\hline
\end{tabular}

According to the expression used in FIS the number of rules for the four attributes can be calculated as:

$$
[3]^{4}=81
$$

Here three is the number of membership function, which is low, medium and high. The output fuzzy decision sets are aggregated into a single fuzzy set and passed to the defuzzifier to be converted into a precise quantity, the $\mathrm{HO}$ factor, which determines whether a $\mathrm{HO}$ is necessary or not.

The range for output function (HO factor) is from zero to one.

From the working flow illustrated in Figure 21, to make a decision to perform the $\mathrm{HO}$ based on RSS, there are two parameters: RSSc for the current cell and RSSn for the neighbouring cell. When RSSc $<$ RSSn the rest of the input parameters are fed to the fuzzy system which computes the HO factor. If this factor is above a predefined threshold (0.7 in our case) the $\mathrm{HO}$ is performed.

Figure 21 Working flow of VHD using fuzzy logic (see online version for colours)

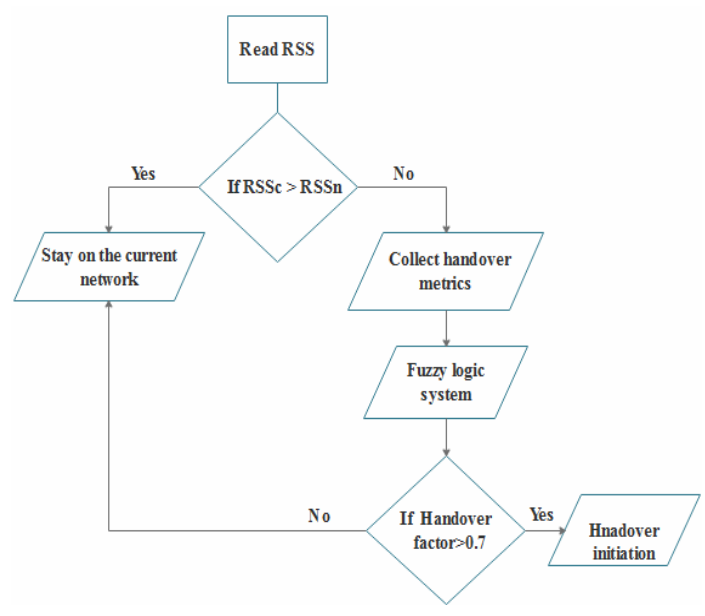

\subsubsection{Modelling and simulation of a fuzzy system}

In this experiment, the model proposed in Figure 10 is used, where the MT moves away from the LTE towards the LTE-A. The objective of the HO from the LTE to LTE-A is to improve the QoS. Fuzzy logic algorithms can be implemented in the $\mathrm{MN}$ as a $\mathrm{HO}$ decision engine to provide rules for decision making. The input parameters (RSS, bandwidth, battery power, and packet loss) are fed into a fuzzifier, which transforms them into fuzzy sets by 
determining the degree to which they belong to each of the appropriate fuzzy sets via membership function. Figure 22, shows the input and output scenario for fuzzy logic.

Figure 22 Input and output scenario for fuzzy logic (see online version for colours)

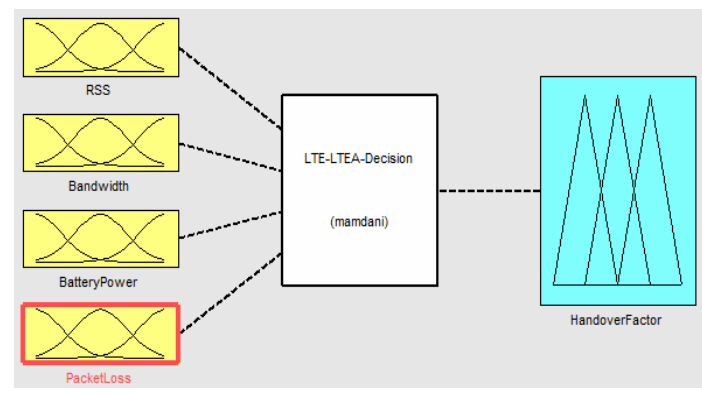

Each of the input parameters is assigned to one of three fuzzy sets, for example, the fuzzy set values for the RSS consist of the linguistic terms: weak, medium, and strong.

The fuzzy set values for the inputs bandwidth, battery power and packet loss are low, medium and high. The fuzzy sets are mapped to corresponding Gaussian membership functions because of their concise notation and having the advantage of being smooth and differentiable at all points. The fuzzy set values for the output decision variable $\mathrm{HO}$ are (lower, low, medium, high and higher).

Figures 23 and 24 illustrate the RSS, the bandwidth, the battery power and packet loss fuzzy sets.

After defining our input and output parameters, the fuzzy sets are fed into a fuzzy inference engine where a set of fuzzy IF-THEN rules is applied to obtain fuzzy decision sets. The output fuzzy decision sets are aggregated into a single fuzzy set and passed to the defuzzifier to be converted into a precise quantity during the final stage of the HO decision. The figure shows some IF-THEN rules that were applied for our fuzzy HO decision algorithm.

Some of The 81 IF-THEN rules are defined as follows and are shown in Figure 25:

- IF RSS is weak, and bandwidth is low, and battery power is high, and packet loss is high, THEN the HO factor is higher.

- $\quad$ IF RSS is strong, and bandwidth is high, and battery power is low, and packet loss is low, THEN the HO factor is lower.

- IF RSS is medium, and bandwidth is medium, and battery power is medium, and packet loss is medium, THEN the HO factor is medium.

If $\mathrm{HO}$ factor is greater than 0.7, the MT initiates $\mathrm{HO}$ to choose the LTE-A; otherwise the MT stays in the current access network (LTE).

Figure 26 illustrates the surface viewer for the fuzzy HO decision in the case of constant battery power and packet loss. As was expected, when the LTE RSS is good and its bandwidth is high, the system chooses to not perform a HO.
Figure 23 Membership function plots, (a) RSS (b) service cost (c) battery power and (d) packet loss (see online version for colours)

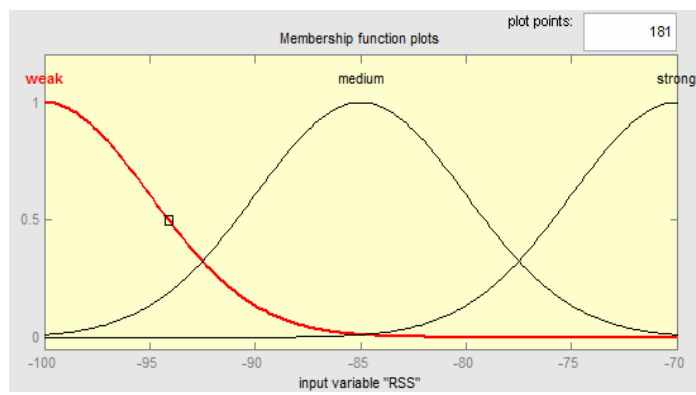

(a)

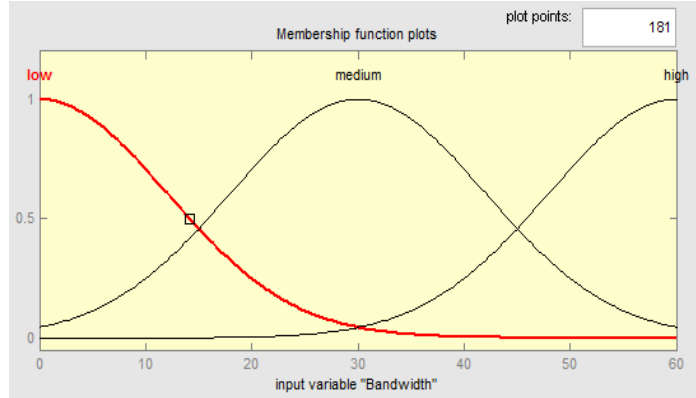

(b)

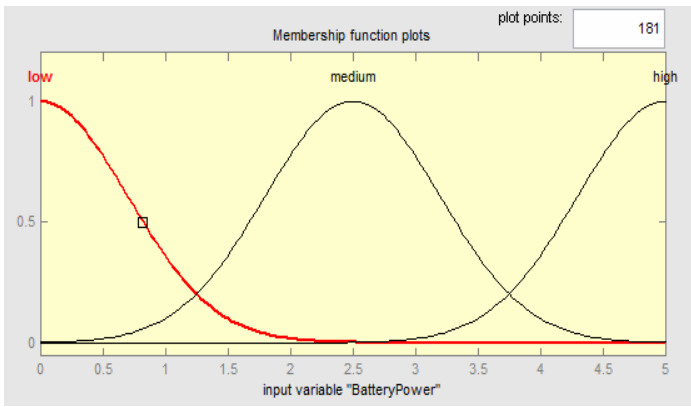

(c)

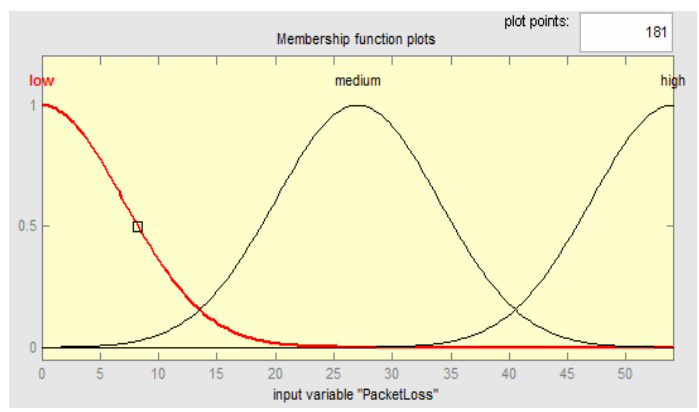

(d) 
Figure 24 Membership function for output variable HO factor (see online version for colours)

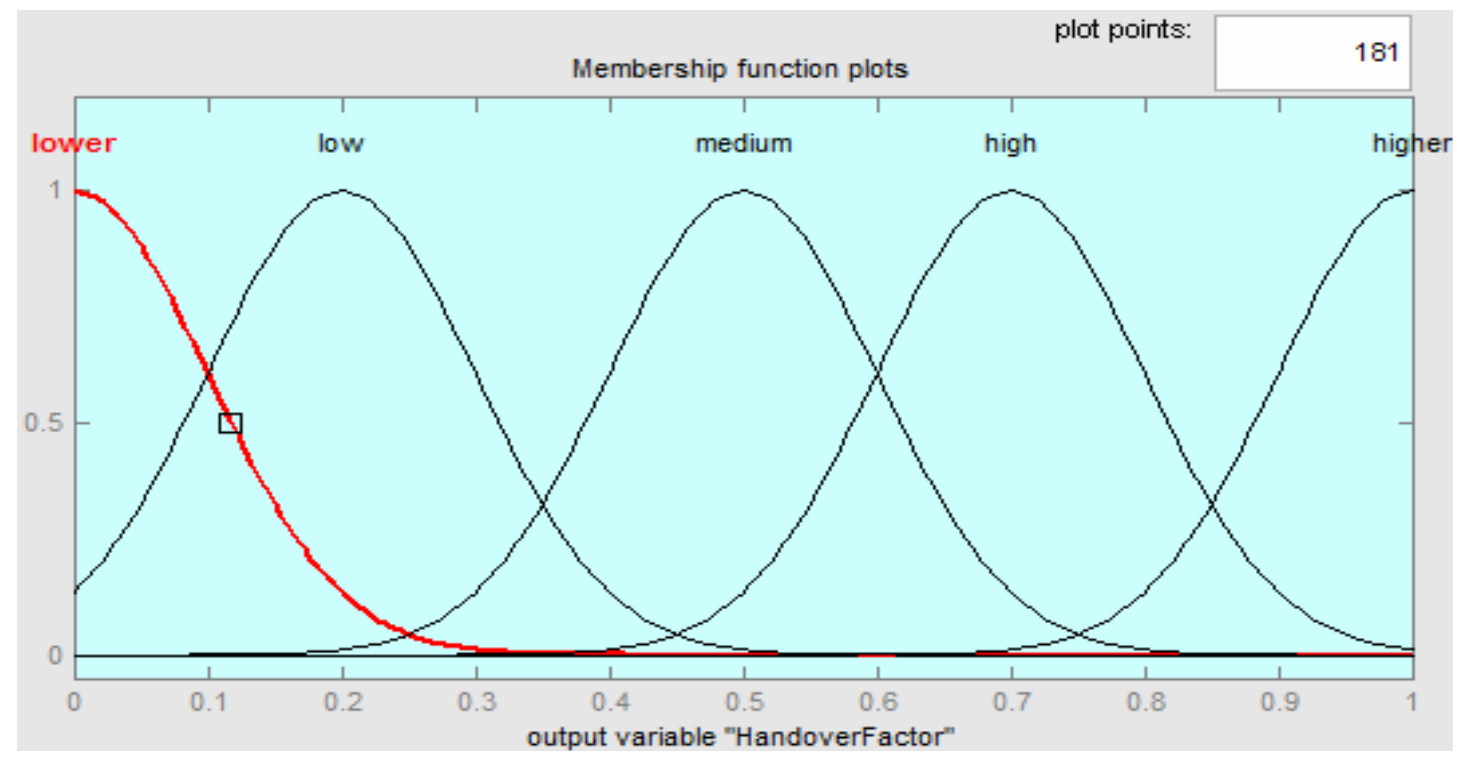

Figure 25 The fuzzy inference rules based on the Mamdani fuzzy inference system (see online version for colours)

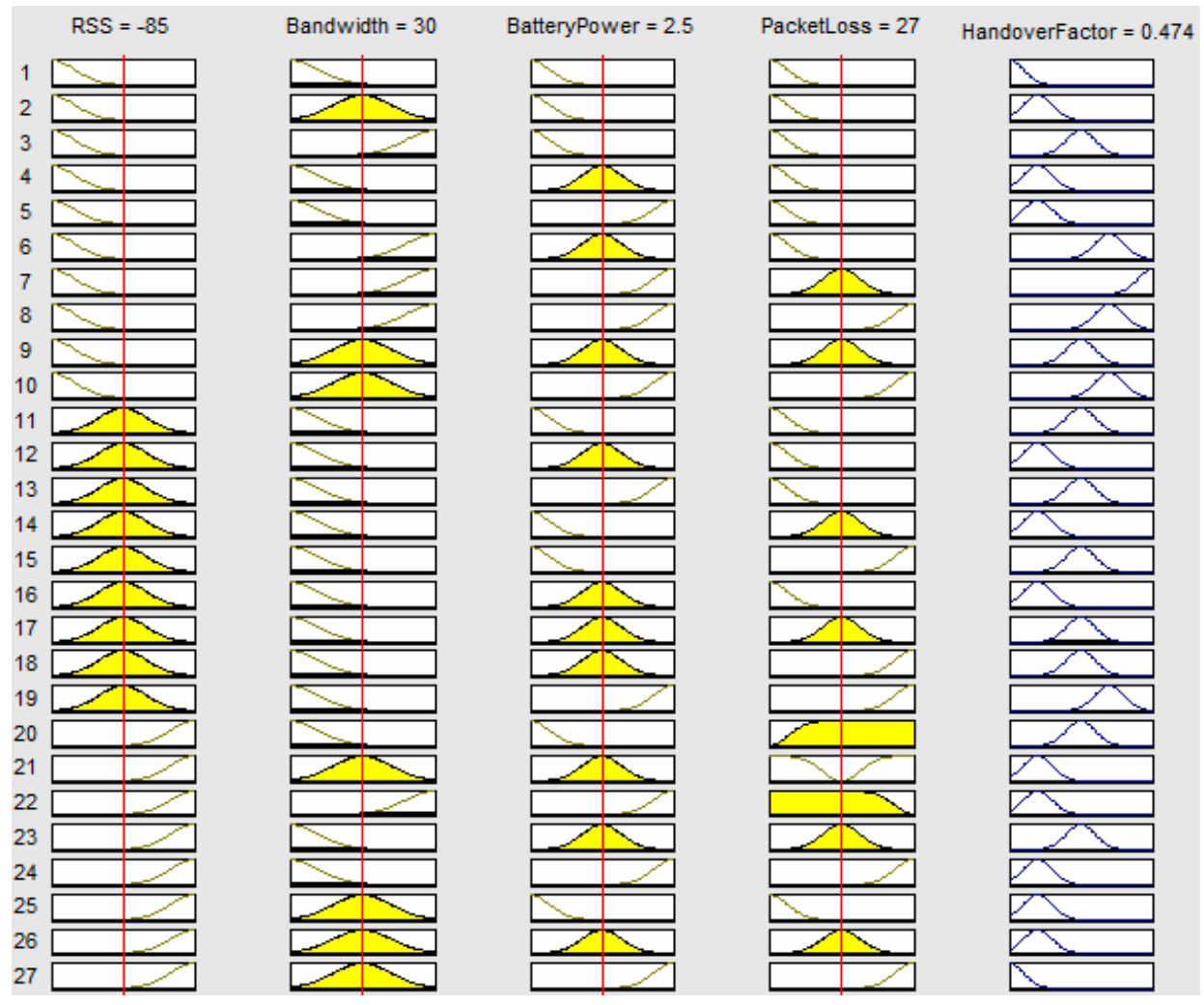


Figure 26 Surface viewer for the fuzzy HO in the case of constant battery power (2.5) and packet loss (30) (see online version for colours)

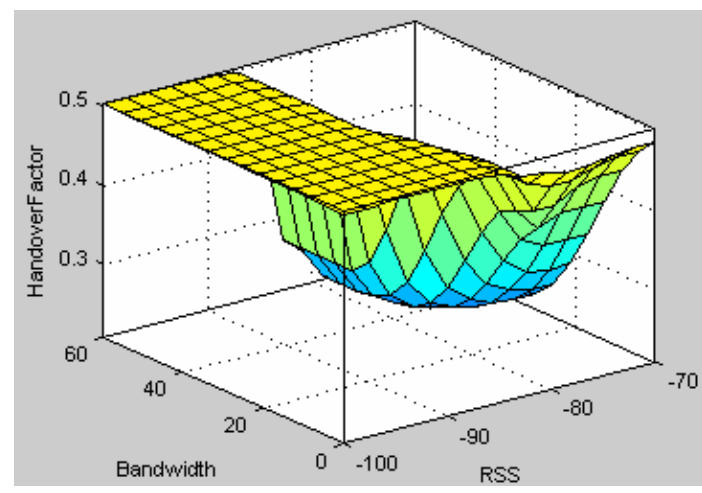

Figure 27 Surface viewer for the fuzzy HO in the case of constant battery power (2.5) and bandwidth (27) (see online version for colours)

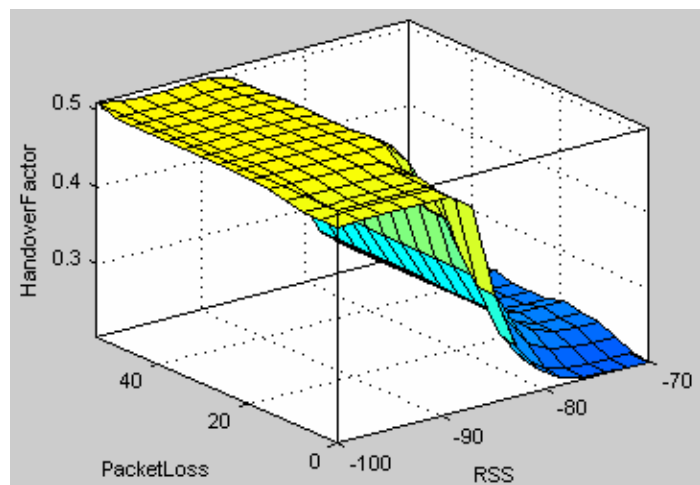

The same behaviour is observed in Figure 27 when the battery power and bandwidth were kept constant, when there is low packet loss the system chooses to not perform a $\mathrm{HO}$.

Figure 28 Surface viewer for the fuzzy HO in the case of constant bandwidth (27) and packet loss (see online version for colours)

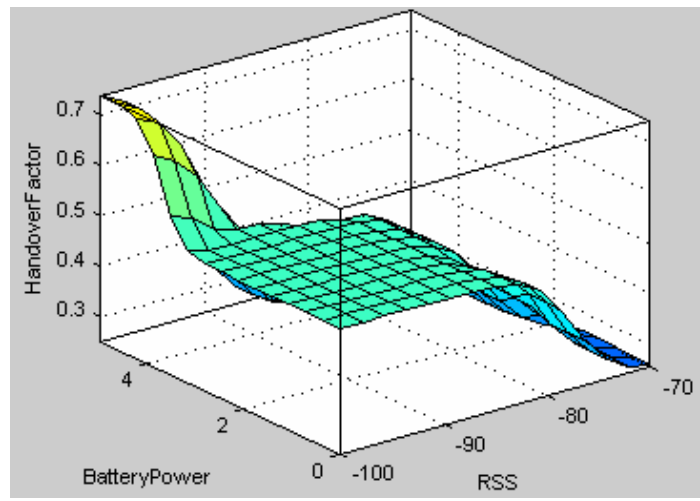

Figure 28 shows the variation of the $\mathrm{HO}$ decision while keeping the bandwidth and packet loss constant. When the signal strength is weak and the battery power increases the fuzzy system tends to prefer the LTE-A and performs HO from LTE to LTE-A.

\subsection{Comparison between vertical HO algorithms}

Figure 29 illustrates the $\mathrm{HO}$ count for each VHD algorithm based on fuzzy logic, RSS, SNR and the traditional approach. It can be seen that the VHD based fuzzy logic results in a substantial reduction in comparison to the other algorithms.

Figure 29 Comparison of HO counts for different approaches (see online version for colours)

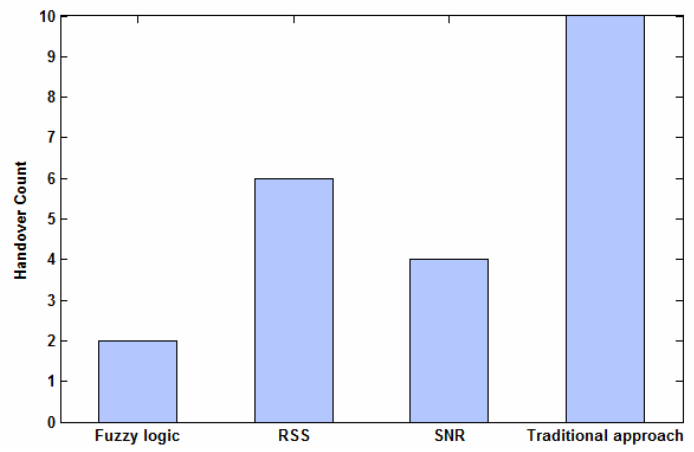

Figure 30 Comparison of power consumption for different approaches (see online version for colours)

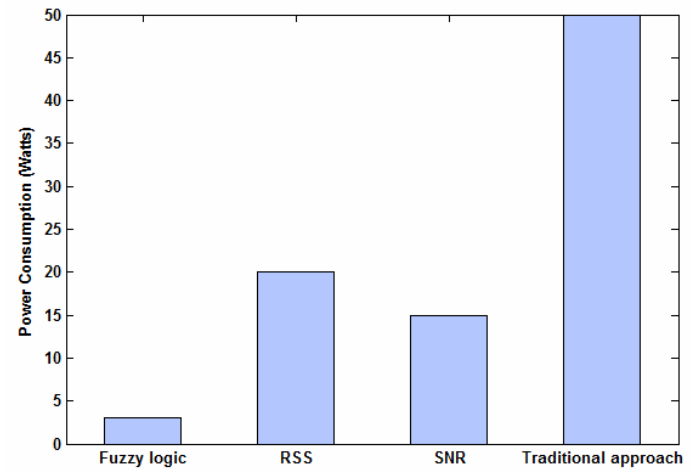

In different networks, the power consumption of the terminal device differs. The users generally choose the network with lower power consumption. The simulation results in Figure 30 show that the VHD based on fuzzy logic leads to lower power consumption compared to RSS, SNR and the traditional approach.

Therefore, it can be concluded that the VHD based on fuzzy logic provides an overall better performance than other vertical $\mathrm{HO}$ algorithms as a consequence of taking into consideration several parameters: 
- RSS: is the most widely used parameter because it is easy to measure and is directly related to the satisfaction of user.

- Bandwidth: system available bandwidth is the major decision factor when terminal choose HO network.

- Battery power: battery power may be a significant factor for $\mathrm{HO}$ in some cases since wireless devices operate on limited battery power. For example, when the battery level decreases, handing off to a network with lower power requirements would be a better decision.

- Packet loss: packet loss is defined as the number of packets lost during the HO. It is the amount of packets that are lost or corrupted and finally cannot reach the desired destinations.

\section{Conclusions and future works}

The main aim of the HO process is to allow the mobile users to roam freely from one mobile network to another whether the network is the same or different. To achieve the load balancing in the different cell $\mathrm{HO}$ it is also necessary to maintain a good radio quality of the link between the mobile users and the serving BS and to minimise the interference level. This paper gives an impression of the main factors which are being affected by the vertical $\mathrm{HO}$ and shows how the RSS for $\mathrm{HO}$ varies according to different networks, especially in the midpoint of the overlapping area, where the network receives a powerful signal and in this case the mobile station carries out a HO.

Therefore our proposed vertical HO scheme guarantees seamless mobility and service continuity by using a concept of RSS in heterogeneous networks. Furthermore, the second algorithm based on fuzzy logic leads to a reduction in the number of HOs and a minimisation of the power consumption as compared to the first algorithm and the existing algorithms. As a follow up for this research work, two topics for future research investigations are suggested. There is a study of SINR and QoS between different types of $\mathrm{HO}$ in heterogeneous networks which asses the energy cost of different vertical handover (VH) schemes, however, designing and implementing new algorithm with energy support in heterogeneous networks would be an interesting line of research.

\section{Acknowledgements}

The research of V. Pla was supported by the Ministry of Economy and Competitiveness of Spain under Grant TIN2013-47272-C2-1-R.

\section{References}

Abubakar, M.M. (2015) 'Intelligent network selection for vertical handover optimization in LTE-advanced', WSEAS Transactions on Communications, Vol. 14, pp.170-179, E-ISSN: 2224-2864

Ahmed, A., Al-Rubiae, H.A. and Al-Qurabi, H.G. (2010) 'Evaluation and comparison of soft and hard handovers in universal mobile telecommunication (UMTS) networks', Journal of Kerbala University, Vol. 8, No. 1 Scientific, pp.232-243.

Alkhayat, I., Kumar, A. and Hariri, S. (2009) 'End-to-end mobility solution for vertical handover between heterogeneous wireless networks', Institute of Electrical and Electronics Engineers (IEEE), pp.750-755.

Athilakshmi, R. and Vijayalakshmi, V. (2015) 'Seamless vertical handoff algorithm for WWANs and WLANs overlay networks', IEEE ICCSP Conference, pp.0425-0429.

Ayyappan, K. and Dananjayan, P. (2008) 'RSS measurement for vertical handoff in heterogeneous network', Journal of Theoretical and Applied Information Technology, pp.990-992.

Bathich, A., Dani Baba, M. and Ibrahim, M. (2012) 'IEEE 802.21 based vertical handover in WiFi and WiMAX networks', IEEE Symposium on Computers \& Informatics (ISCI).

Bchini, T., Tabbane, N., Tabbane, S., Chaput, E. and André-Luc, B. (2010) 'Fuzzy logic based layers 2 and 3 handovers in IEEE 802.16e network', Computer Communications, Vol. 33, pp.2224-2245.

Benaatou, W. and Latif, A. (2014) 'Study of the vertical handover in heterogeneous networks and implement based on opnet', International Journal of Computer, Electrical, Automation, Control and Information Engineering, Vol. 8, No. 8, pp.1435-1439.

Bin, M., Xiaofeng, L. and Xianzhong, X. (2013) 'Vertical handoff algorithm based on type-2 fuzzy logic in heterogeneous networks', Journal of Software, November, Vol. 8, No. 11, pp.936-2942.

Bouali, T. and Senouci, S.M. (2016) 'A fuzzy logic-based communication medium selection for QoS preservation in vehicular networks', IEEE ICC 2016 - Next-Generation Networking and Internet Symposium.

Busanelli, S., Martal, M., Ferrari, G. and Spigoni, G. (2011) Vertical Handover between WiFi and UMTS Networks: Experimental Performance Analysis, Vol. 2, No. 1, pp.75-95.

Chinnappan, A. and Balasubramanian, R. (2016) 'Complexity-consistency trade-off in multi-attribute decision making for vertical handover in heterogeneous wireless networks', IET Journals and Magazines, Vol. 5, No. 1, pp.13-21.

Datta, P. and Kaushal, S. (2015) 'Fuzzy logic-based handover in 3GPP LTE network, intelligent computing and applications', Advances in Intelligent Systems and Computing, Vol. 343, pp.213-222, Springer.

Divya, G. and Suman, D. (2015) 'Effective network handovers using fuzzy inference for heterogeneous mobility management', International Journal of Science, Engineering and Technology Research (IJSETR), July, Vol. 4, No. 7, pp.2379- 2383. 
Farnaz, F., Seyed, S. and Chun, R. (2014) 'A fuzzy logic approach for quality of service quantification in wireless and mobile networks', 10th IEEE International Workshop on Performance and Management of Wireless and Mobile Networks.

Gavali V.S. and Patil, J.K. (2015) 'A study of RSS based vertical handover decision algorithms', International Journal of Engineering Research \& Technology (IJERT), Vol. 4, No. 2, pp.825-827.

Hamada, R.A., Ali, H.S. and Abdalla, M.I. (2014) 'SIP-based mobility management for LTE-WiMax-WLAN interworking using IMS architecture', International Journal of Computer Networks (IJCN), Vol. 6, No. 1, pp.1-14.

Joon, R., Sandeep and Chadha, M.S. (2012) 'Analysis of WIMAX handover', International Journal of Soft Computing and Engineering (IJSCE), July, Vol. 2, No. 3, pp.476-479, ISSN: 2231-2307.

Karanjekar, H. and Agrawal, A. (2014) 'Review on vertical handover techniques among heterogeneous networks', Int. J. Advanced Networking and Applications, Vol. 5, No. 5, pp.2066-2069, ISSN: 0975-0290.

Kassar, M., Kervella, B. and Pujolle, G. (2008) 'An intelligent handover management system for future generation wireless networks', EURASIP Journal on Wireless Communications and Networking, Vol. 2008, Article ID 791691, 12pp., DOI: 10.1155/2008/791691.

Kassar, M., Kervella, B. and Pujolle, G. (2008) 'An overview of vertical handover decision strategies in heterogeneous wireless networks', Computer Communications, January, Vol. 31, pp.2607-2620.

Kunarak, S. and Suleesathira, R. (2010) 'Predictive RSS with fuzzy logic based vertical handoff algorithm in heterogeneous wireless networks', International Conference on Advanced Technologies for Communications (ATC).

Kunarak, S. and Suleesathira, R. (2013) 'Algorithmic vertical handoff decision and merit network selection across heterogeneous wireless networks', Wseas Transactions on Communications, Vol. 12, No. 1, pp.1-12.

Lin, C. and Hui, L. (2016) 'An MDP-based vertical handoff decision algorithm for heterogeneous wireless networks', IEEE Wireless Communications and Networking Conference (WCNC) - Track 3 - Mobile and Wireless Networks, pp.1-6, ISSN: 1558-2612.

Ling, J., Hui, Z., Long-Xiang, Y. and Hong-Bo, Z. (2014) 'An adaptive vertical handoff algorithm based on UMTS and WLAN', Telkomnika Indonesian Journal of Electrical Engineering, Vol. 12, No. .5, pp.3943 -3954.

Liu, B., Martins, P. and Bertin, P. (2010) 'Cross-layer design of the inter-RAT handover between UMTS and WiMax', EURASIP Journal on Wireless Communications and Networking, Article ID 763614, 13pp., DOI: 10.1155/2010/ 763614.

Liu, H., Maciocco, C. and Kesavan, V. (2008) 'A smart triggering scheme to reduce service interruption during heterogeneous handovers', IEEE International Conference on Dependable Systems and Networks with FTCS and DCC.

Manjaiah, M. and Payaswini, P. (2013) 'A review of vertical handoff algorithms based on multi attribute decision method', International Journal of Advanced Research in Computer Engineering and Technology, Vol. 2, No. 6, pp.2005-2008.

Manoj, S. (2015) 'Fuzzy logic tool for imprecise information in wireless communication-another perspective', International Journal of Research in Management, Science and Technology, Vol. 3, No. 1, pp.57-61, E-ISSN: 2321-3264.
Mardini, W. and Al-Ghadi, M.Q. (2012) 'Distance-based scheme for vertical handoff in heterogeneous wireless networks', Journal of Emerging Technologies in Web Intelligence, Vol. 4, No. 1, pp.67-76.

Miyim, A.M., Ismail, M. and Nordin, R. (2014) 'Vertical handover solutions over LTE-advanced wireless networks: an overview', Wireless Personal Communications, August, Vol. 77, No. 4, pp.3051-3079.

Miyim, A.M., Ismail, M., Nordin, R. and Taha, I.M. (2013) 'Technique for cross-layer vertical handover prediction in 4G wireless networks', 4th International Conference on Electrical Engineering and Informatics, ICEEI, Vol. 11, pp.114-121.

Neeraj, K. and Chilamkurti, N. (2012) 'Agent assisted mobility and load aware fast handoff scheme in wireless mesh networks', Int. J. Internet Protocol Technology, Vol. 7, No. 1, pp.26-38.

Nithyanandan, L. and Parthiban, I. (2012) 'Vertical handover in WLAN-WIMAX-LTE heterogeneous networks through gateway relocation', International Journal of Wireless and Mobile Networks (IJWMN), August, Vol. 4, No. 4, pp.203-215.

Padmalaya, N. and Anurag, D. (2016) 'A fuzzy logic-based clustering algorithm for WSN to extend the network lifetime', IEEE Sensors Journal, January, Vol. 16, No. 1, pp.137-144.

Parul, D. and Sakshi, K. (2014) 'Fuzzy logic-based handover in 3GPP LTE network', Proceedings of the International Conference on ICA, Intelligent Computing and Applications, Advances in Intelligent Systems and Computing, pp.22-24.

Qasim, N. and Sheikh, M.S. (2011) 'Vertical handover in wireless overlay networks with quality of service factors', World Congress on Engineering, Vol. II, pp.6-8.

Reddy, S., Vamshidhar, R. and Sanjay, D.R. (2013) 'Vertical handoff decision algorithms in integrated heterogeneous networks', International Journal of Energy, Information and Communications, April, Vol. 4, No. 2, pp.36-40.

Roy, S.D. and Vamshidhar, R.R. (2014) 'Signal strength ratio based vertical handoff decision algorithms in integrated heterogeneous networks', Wireless Personal Communications, Vol. 77, No. 4, pp.2565-2585.

Savitha, K. and Chandrasekar, D.R.C. (2011) 'Network selection using topsis in vertical handover decision schemes for heterogeneous wireless networks', IJCSI International Journal of Computer Science Issues, Vol. 8, Nos. 3-2, pp.400-406.

Shayea, I., Ismail,M. and Rosdiadee, N. (2012) 'Advanced handover techniques in LTE-advanced system', International Conference on Computer and Communication Engineering (ICCCE 2012), pp.74-79.

Stevens-Navarro, E. and Wong, V. (2006) 'Comparison between vertical handoff decision algorithms for heterogeneous wireless networks', IEEE VTC, Vehicular Technology Conference.

Tong, D., Jia, Z., Xizhong, Q., Cao, C. and Chang, C. (2014) 'An optimal Savitzky-golay filtering based vertical Hando_ algorithm in heterogeneous wireless networks', Journal of Computers, November, Vol. 9, No. 11, pp.2685-2690.

Ulvan, R., Bestak, R. and Ulvan, M. (2010) 'Handover scenario and procedure in LTE-based Femtocell networks', The Fourth International Conference on Mobile Ubiquitous Computing, Systems, Services and Technology, pp.213-218. 
Vandana, D. and Surendra, S. (2015) 'Comparison of fuzzy rule based vertical handover with TOPSIS and received signal strength based vertical handover algorithms', IOE Graduate Conference, pp.105-111.

Vegni, A.M., Tamea, G. and Inzerilli, T. (2009) 'A combined vertical handover decision metric for QoS enhancement in next generation networks', IEEE International Conference on Wireless and Mobile Computing, Networking and Communications.

Yadav, J. and Mehandia, B. (2014) 'Handover performance in WIMAX mobile networks', International Journal of Advanced Research in Computer and Communication Engineering, August, Vol. 3, No. 8, pp.7696-7699.
Yang, K., Gondal, I., Qiu, B. and Dooley, L.S. (2007) 'Combined SINR based vertical handoff algorithm for next generation heterogeneous wireless networks', IEEE Global Telecommunications Conference.

Yujae, S., Peng-Yong, K. and Youngnam, H. (2014) 'Power-optimized vertical handover scheme for heterogeneous wireless networks', IEEE Communications Letters, February, February, Vol. 18, No. 2, pp.277-280. 